\title{
Wall shear stress in a subject specific human aorta - Influence of fluid-structure interaction
}

Jonas Lantz, Johan Renner and Matts Karlsson

\section{Linköping University Post Print}

N.B.: When citing this work, cite the original article.

Original Publication:

Jonas Lantz, Johan Renner and Matts Karlsson, Wall shear stress in a subject specific human aorta - Influence of fluid-structure interaction, 2011, International Journal of Applied Mechanics, (3), 4, 759-778.

http://dx.doi.org/10.1142/S1758825111001226

Copyright: World Scientific Publishing

http://www.worldscinet.com/

Postprint available at: Linköping University Electronic Press

http://urn.kb.se/resolve?urn=urn:nbn:se:liu:diva-71720 


\title{
WALL SHEAR STRESS IN A SUBJECT SPECIFIC HUMAN AORTA - INFLUENCE OF FLUID-STRUCTURE INTERACTION
}

\author{
JONAS LANTZ* JOHAN RENNER, MATTS KARLSSON \\ Department of Management and Engineering \\ Linköping University \\ SE-581 83 Linköping \\ Sweden \\ jonas.lantz@liu.se
}

Received date

Vascular wall shear stress (WSS) has been correlated to the development of atherosclerosis in arteries. As WSS depends on the blood flow dynamics, it is sensitive to pulsatile effects and local changes in geometry. The aim of this study is therefore to investigate if the effect of wall motion changes the WSS or if a rigid wall assumption is sufficient. Magnetic resonance imaging (MRI) was used to acquire subject specific geometry and flow rates in a human aorta, which were used as inputs in numerical models. Both rigid wall models and fluid-structure interaction (FSI) models were considered, and used to calculate the WSS on the aortic wall. A physiological range of different wall stiffnesses in the FSI simulations was used in order to investigate its effect on the flow dynamics. MRI measurements of velocity in the descending aorta were used as validation of the numerical models, and good agreement was achieved. It was found that the influence of wall motion was low on time-averaged WSS and oscillating shear index, but when regarding instantaneous WSS values the effect from the wall motion was clearly visible. Therefore, if instantaneous WSS is to be investigated, a FSI simulation should be considered.

Keywords: computational fluid dynamics; wall deformation; windkessel model; pressure wave; magnetic resonance imaging

\section{Introduction}

The development of atherosclerosis and its flow-dependence is one of the main reasons for simulating the flow in the aortic system. Atherosclerotic lesions has been found to develop in the vicinity of branching arteries and strong curvature, where disturbed flow patterns are present [Ku et al., 1985; Malek et al., 1999]. In these areas low and/or oscillating wall shear stress (WSS) have been established as predictors of increased risk for the development atherosclerotic plaques [Moore et al., 1994; Irace et al., 2004].

In recent years there has been a great emphasis on fluid-structure interaction (FSI) modelling of arterial blood flow. The topics investigated range from heart 
valves [Cheng et al., 2003, 2004; Dahl et al., 2010; Simon et al., 2010], to estimation of wall stresses and strains in either healthy subjects [Gao et al., 2006b; McGregor et al., 2007] or in pathological cases such as aneurysms [Isaksen et al., 2008; Li and Kleinstreuer, 2005a,b; Scotti et al., 2008; Kelly and O'Rourke, 2010; Xenos et al., 2010; Tan et al., 2009]. There have also been attempts to estimate the WSS on the arterial surface [Jin et al., 2003; Gao et al., 2006a; Shahcheraghi et al., 2002]. Some advantages with FSI-simulations are that the arterial wall movement due to the pulsatile blood flow is resolved, and the pressure wave can be computed. The drawback is that it is very computationally expensive compared to a simulation where the wall is considered rigid, as the coupling between the fluid and solid domain increases model complexity.

Also, assumptions of patient specific material parameters must be made, such as wall elasticity and the tissue surrounding the blood vessel. There is ongoing research trying to circumvent this by measuring the wall motion and then prescribe it in the CFD model, see e.g. [Jin et al., 2003] for the case of the ascending aorta and [Dempere-Marco et al., 2006] for the case of intracranial aneurysms. The timeresolution of those measurements are on the order of 20 frames per second, and to get a smooth transition from one time step to another, a transformation from one image to the next needs to be done. If there are sudden movements between two images this may not be accounted for. On the other hand, in a FSI simulation the time resolution is arbitrary and the stress in the wall can be resolved.

WSS is a very sensitive parameter as it is based on the velocity profile at the wall, and the deformation of the arterial wall might have a large impact on it. It was therefore decided to investigate if WSS is dependent on the wall movement, or if rigid-wall simulations, which are cheaper to run, can be considered.

In this article, MRI-acquired geometry and flow rates of a subject specific human aorta are used as input to different simulation models, in order to determine the local flow dynamics. Measured velocity in the ascending aorta were used as an inlet boundary condition while measured velocity in the descending aorta were used to validate the simulation results. A comparison between FSI and rigid-wall models was performed in order to investigate the rigid-wall assumption.

\section{Method}

The workflow of estimating subject specific WSS in this work includes MRI measurements of geometry and blood flow in subjects, segmentation of the MRI geometry into CAD surfaces, creation of computational meshes, simulation setup (including physiological boundary conditions and constraints), solving the simulation, and post-processing the results. In this section the methodology is presented, with focus on the simulation part. 


\subsection{MRI acquisition and segmentation}

Geometric and flow data were acquired using a 1.5 T Philips Achieva MRI scanner. The complete aorta was obtained within a breath hold and the 3D volume data was reconstructed to a resolution of $0.78 \times 0.78 \times 1.00 \mathrm{~mm}^{3}$. To retrieve a physiological inlet velocity profile for the fluid simulation, time-resolved aortic flow rates were obtained by performing throughplane $2 \mathrm{D}$ velocity MRI acquisition placed supracoronary and perpendicular to the flow direction and was reconstructed to 40 time-frames per cardiac cycle with a spatial resolution of $1.37 \times 1.37 \mathrm{~mm}^{2}$. The throughplane where the velocity was measured is indicated as section A-A in Fig. 1. Aortic geometry from MRI images were extracted with a 3D level set algorithm and used for segmentation. In the image-volume one or several seed points/regions are defined and an implicit surface was allowed to expand outwards to the edge of the object. The algorithm was implemented into a cardiac image analysis software package [Heiberg et al., 2010]. The segmentation software gave a STL-representation of the inner aortic wall which set the boundary for the fluid domain [Svensson et al., 2006].
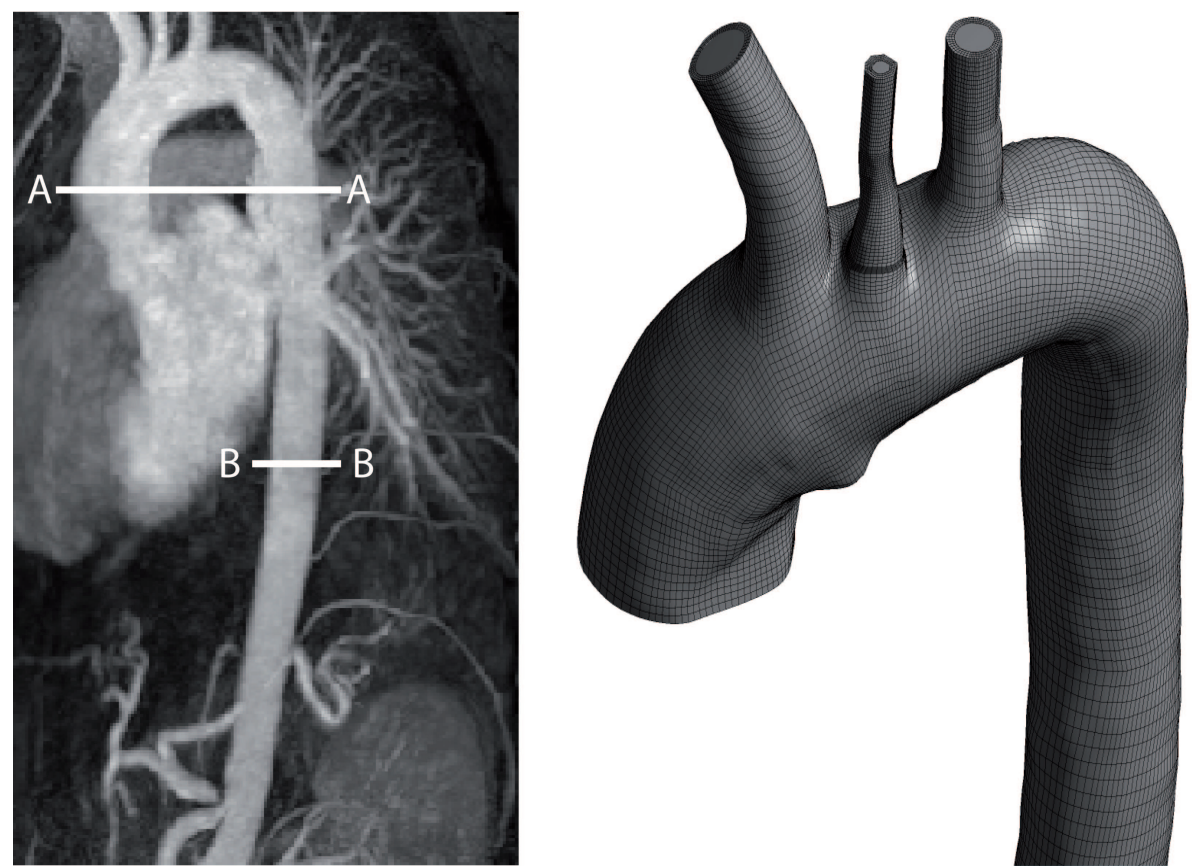

Fig. 1. Left: Maximum Intensity Projection (MIP) MRI of the aorta. Section A-A shows where flow profiles were measured and where the numerical model where cut in the ascending aorta. Also, in the descending aorta, section A-A indicates where the numerical model is validated with MRI measurements. Section B-B show where the model ends in the thoracic aorta and where the impedance pressure boundary condition is set. Right: the computational mesh of the solid domain used in the FSI simulations. 


\subsection{Numerical models}

In this study, two-way fully coupled FSI simulations were carried out, solving the solid and fluid problem in a sequential manner and coupled through dynamic and kinematic interfaces. The dynamic interface meant that the pressure and shear load on the fluid side balanced the traction of the solid side, whereas the kinematic interface meant that the velocity of the solid had to match the velocity of the fluid.

\subsubsection{Meshes}

The segmented geometry obtained from the MRI measurement was cut in the ascending aorta at section A-A and in the thoracic aorta at section B-B, see Fig. 1. The brachiocephalic, left common carotid, and left subclavian arteries (Fig 1, upper three branches from left to right) were extended $30 \mathrm{~mm}$ to minimize numerical problems at the outlets. No other arteries leaving the aorta were included in the model. High quality hexahedral computational meshes were constructed in ANSYS ICEM CFD 12.0 (ANSYS Inc. Canonsburg, PA, USA) and used in both the fluid and the solid geometries. The mesh quality metric in ICEM has a range from -1 to 1 , where -1 is unacceptable, 1 is perfect, and 0.2 is usually considered as an acceptable threshold value. The quality is a weighted diagnostic between Determinant, Max Orthogls and Max Warpgls, and the minimum of the 3 diagnostics is presented as the quality of the mesh. The Determinant is the ratio of the smallest determinant of the Jacobian matrix divided by the largest determinant of the Jacobian matrix, where a value of 1 would indicate a perfectly regular mesh element, while a negative value would indicate an inverted element. Max Orthogls is the calculation of the maximum deviation of the internal angles from 90 degrees, and Max Warpgls is the warp of a quadrilateral faces of a hex element. For more details, see [ANSYS Inc., 2010b]. The stability and robustness of the simulations proved to be very sensitive to mesh quality, and therefore a lot of effort was put into making the mesh as good as possible. In total, $97 \%$ of the mesh elements had a quality above 0.7 and remaining $3 \%$ had a quality above 0.4 . To resolve the boundary layer, the fluid mesh had 10 prism layers adjacent to the wall, which grew exponentially until the thickness of the last layer matched the core mesh size. The dimensionless wall distance $y^{+}$is important to check, as it is a measure of the near-wall resolution and also determines how the solver handles the wall (see next section). The $y^{+}$ values changes during a cardiac cycle due to the fluid motion. For highly accurate simulations where the boundary layer treatment is important a $y^{+}$in the order of 1-2 is needed, and in these simulations it was found to to have a maximum value of 1.5 during the cardiac cycle.

Mesh independency tests were carried out with $0.5 \mathrm{MC}$ (million cells), 2.8 MC, and $4.8 \mathrm{MC}$ and it was found that the $u, v$, and $w$ velocity profiles in the descending aorta were identical (within $5 \%$ ) for all three meshes. To save computational power, the $0.5 \mathrm{MC}$ mesh was therefore chosen. The solid mesh had roughly 65000 elements, and the wall thickness was divided into 3 elements. The element concentration was 
higher in areas were large deformations were found to occur. To find these areas an iterative process was employed: a simulation was run and in areas were large displacement occured the element concentration was increased. Then the simulation was rerun and the displacement was compared to the smaller mesh. When no significant difference in deformation could be found, the solid mesh was said to be mesh independent.

\subsubsection{Fluid model}

The fluid was set to resemble blood with a constant viscosity of $2.56 \mathrm{e}-3 \mathrm{~Pa}$ s (i.e. Newtonian fluid) [Wells and Merril, 1962] and a density of $1080 \mathrm{~kg} / \mathrm{m}^{3}$ [Kenner, 1989]. From the velocity profiles in the MRI measurements, the Reynolds number based on inlet diameter, ranged from 200 at late diastole to 7500 at peak systole, with a mean of 1600 . Therefore, the $k-\omega$ based Shear Stress Transport (SST) turbulence model was used, which accounts for the transport of turbulent shear stress and gives reasonable predictions of the onset and amount of flow separation under adverse pressure gradients [ANSYS Inc., 2010a]. The SST turbulence model solves the $k-\omega$ model in the near wall region and the $k-\epsilon$ model in the bulk flow, with blending functions to ensure a smooth transition between the two models. The automatic near-wall treatment method available in CFX was used to model the flow near the wall. This method switches between wall-functions and a lowReynolds number near wall formulation depending on mesh resolution. The wallfunction approach uses empirical correlations near the wall without resolving the boundary layer. On the other hand, the low-Reynolds number approach resolves the details of the boundary layer profile, increasing accuracy of the boundary layer. As the boundary layer mesh is well resolved, the near-wall treatment is used, instead of wall-functions.

Velocity profiles were measured with MRI in a plane in both the ascending and descending aorta (see section A-A in Fig. 1), enabling the use of physiological correct inlet velocity profiles. A sensitivity study showed that the time step needed to resolve the flow accurately was $0.005 \mathrm{~s}$, but only 40 time frames per cardiac cycle (about 1 frame every $0.03 \mathrm{~s}$ ) was measured with the MRI. Therefore, linear interpolation of the velocity profiles was performed to get a smooth inlet boundary condition. To represent initial disturbances in the flow, a turbulence intensity of $5 \%$ was specified on the inlet. From the measured flow rates, the flow leaving the arteries in the aortic arch could be deducted, as the difference between the ascending and descending flow rates. Mass flow rates were specified on each of the three arteries in the aortic arch; the difference between ascending and descending flow times a scale factor were used. The scale factors were based on cross-sectional area and were: $10 / 16,1 / 16,5 / 16$, for the brachiocephalic, left common carotid, and left subclavian artery, respectively.

On the lower outlet in the thoracic aorta an impedance boundary condition was imposed to get a realistic pressure wave profile. A three-element Windkessel 
J. Lantz et al.

model was used, which describes the relationship between the aortic outflow and aortic pressure. The Windkessel model is a lumped model, and is capable of defining physiologically relevant pressure waves [Westerhof et al., 2009; Stergiopulos et al., 1999]. The model can be viewed analogously to an electrical circuit, where the current represent the flow rate and the voltage difference the drop in pressure.

This is modelled as a resistance $R_{1}$ connected to a resistance $R_{2}$ in parallel to a capacitance $C_{s}$, see Fig. 2. The resistance $R_{1}$ is a measure of the characteristic resistance of the artery, while $R_{2}$ models the peripheral resistance, which can be seen as the resistance the blood encounters when flowing through the arterial tree from larger to smaller arteries. The capacitance $C_{s}$ is a model of the total systemic arterial compliance [Grant and Paradowski, 1987].

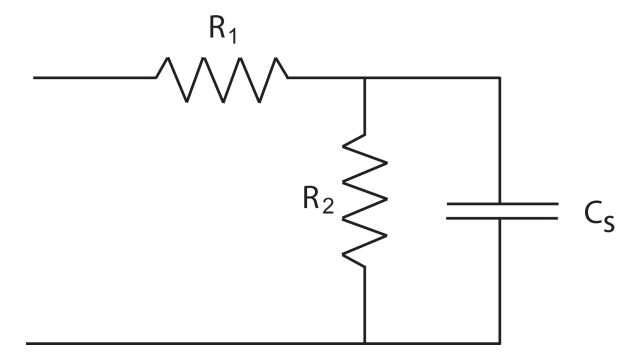

Fig. 2. Electrical analogy of a 3-element Windkessel model.

For the model in Fig. 2, the pressure-flow rate relationship becomes:

$$
\begin{array}{r}
P(t)=P_{c}(t)+R_{1} \dot{Q}(t) \\
\dot{Q}(t)=C_{s} \frac{d P_{c}(t)}{d t}+\frac{P_{c}(t)}{R_{2}}
\end{array}
$$

where $R_{1}, R_{2}$, and $C_{s}$ have been defined earlier and $P(t), P_{c}(t)$ and $\dot{Q}(t)$ are the total pressure, capacitance pressure, and flow rate, respectively. The equation system was solved by approximating the time derivative with a forward difference. Following [Beulen et al., 2009], the peripheral resistance $R_{1}$ was obtained by modelling minimal reflections at the outlet, and the $R_{2}$ and $C_{s}$ parameters were then adjusted to obtain a physiological pressure drop. The values of $R_{1}, R_{2}$ and $C_{s}$ used in the model were $8.3 \mathrm{e} 9 \mathrm{~kg} \mathrm{~m}^{-4} \mathrm{~s}^{-1}, 9.5 \mathrm{e} 9 \mathrm{~kg} \mathrm{~m}^{-4} \mathrm{~s}^{-1}$, and $1.6 \mathrm{e}-10 \mathrm{~m}^{4} \mathrm{~s}^{2} \mathrm{~kg}^{-1}$, respectively.

\subsubsection{Wall model}

The solid domain was modelled as a nearly incompressible, linear elastic material with an isotropic Young's modulus. The density of the solid was set to $1080 \mathrm{~kg} / \mathrm{m}^{3}$ and the Poisson's ratio to 0.499, which are commonly used values [Bathe et al., 1999; McGregor et al., 2007; Wolters et al., 2005]. As the wall stiffness is a subject 


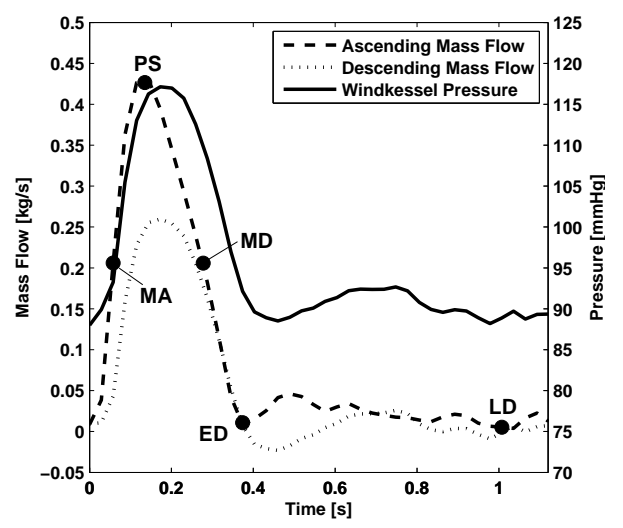

Fig. 3. Measured mass flow rates in ascending and descending aorta (left y-axis), and the resulting Windkessel pressure profile in the thoracic aorta (right y-axis). Black dots indicate when some of the results were evaluated; MA stands for max acceleration, PS peak systole, MD max deceleration, ED early diastole, and LD late diastole, respectively.

specific parameter, an universal value does not exist. A number of authors have used a Young's modulus close to $1 \mathrm{MPa}$, see e.g. [Li and Kleinstreuer, 2005b; Gao et al., 2006a], and it was therefore decided to use $1 \mathrm{MPa}$ in this study. To enhance the effect of the FSI, two lower wall stiffnesses were also considered: 0.5 and $0.75 \mathrm{MPa}$, which are also in the physiological range. The three FSI models are from here on referred to as FSI E 0.5 , FSI E 0.75, and FSI E 1.0, respectively. The wall thickness is usually not constant; it can vary in both axial and circumferential direction. However, without own measurements of the thickness, a uniform thickness of $1 \mathrm{~mm}$ was assumed and offseted from the measured inner wall. This value is considered low, but still physiological, according to CT-scans of normal healthy men [Jeltsch et al., 2009]. The geometry was constrained in axial direction at the in- and outlets, but allowed to move in radial and circumferential directions at all locations. The tissue surrounding the aorta was expected to have a damping effect on the aortic wall movement, and this effect was taken into account by applying a linear elastic support boundary condition. The elastic support is based on a stiffness foundation, which is defined as the pressure required to produce a unit normal deflection of the foundation of the support. Throughout the simulations, a foundation stiffness of $75 \mathrm{mmHg} / \mathrm{mm}$ was used, which means that if $75 \mathrm{mmHg}$ of pressure is applied to the wall, it will be displaced $1 \mathrm{~mm}$. If the pressure decreases, the wall will move back accordingly. This represents a simple approximation of the influence of the complex surroundings of the aorta, since the actual relationship is rather difficult to determine. It also helps to remove the high-frequency modes of the structural deformation [Takizawa et al., 2010]. 


\subsubsection{Simulation}

The calculations were performed with ANSYS CFX 12.0 for the fluid simulations and ANSYS Mechanical for the solid simulations. The FSI-algorithm is iteratively implicit, meaning that an implicit result is found by iterating the loads transferred at the interfaces for each time step. The time steps were divided into a number of coupling iterations called staggers, and for each stagger iteration, CFX passed the total force on the interface wall to ANSYS Mechanical, which in turn passed the resulting total mesh deformation back to ANSYS CFX. The stagger iterations were repeated until all field equations were converged and then an advance in time was executed. A maximum root-mean-square (RMS) residual of $10^{-6}$ was defined for the fluid simulation, and a maximum RMS residual of $10^{-3}$ for the solid simulation to ensure a well-converged solution. For each time step an upper limit of 30 stagger iterations was set but the solution always converged before that limit. Domain imbalances were converged below $0.5 \%$ and for the interface quantities on the FSIinterface, the convergence criteria was $10^{-4}$ while the convergence criteria for mesh displacement was $10^{-5}$. Independence tests were carried out on all of the convergence criteria to ensure an accurate solution. Temporal discretization was performed with a second order backward Euler scheme and the spatial discretization used second order differencing.

Ideally, the MRI measurements would be performed at different times during the cardiac cycle, yielding a specific geometry for each time point. However, the measurements are an average of the geometry during several cardiac cycles. As the systolic phase is only one third of the pulse while the diastolic phase is two thirds, it is reasonable to assume that the acquired geometry is diastolic-dominant.

In order to investigate the importance of deforming walls, both FSI and regular CFD simulations with rigid walls were performed. In total, five simulations were run, three FSI with different wall stiffnesses (Young's modulus of 0.5, 0.75, and 1.0 $\mathrm{MPa}$ ) and two rigid wall simulations. The rigid wall simulations were taken from the resulting geometry of the 1.0 MPa FSI simulation at peak systole (PS) and at late diastole (LD). This corresponds to two geometrical approximations of imaginary snapshot MRI measurements at those two times. The reason peak systole and late diastole were chosen is because they represents the two extremes in terms of deformation and domain volume. The three FSI simulations will have different degrees of deformation, which is shown in Fig. 4.

Seven cardiac cycles were simulated and results are only taken from the last cycle. The last three cardiac cycles were identical in terms of flow and displacement, indicating that the results were independent of initial and transient effects. Due to the strong convergence criteria in the FSI simulations, one cardiac pulse took about one week to simulate on an eight-core Intel Xeon E5520 machine with 32 GB of RAM. The simulations were run on the Linux clusters Neolith and Kappa at National Supercomputer Centre (NSC), Linköping, Sweden. 


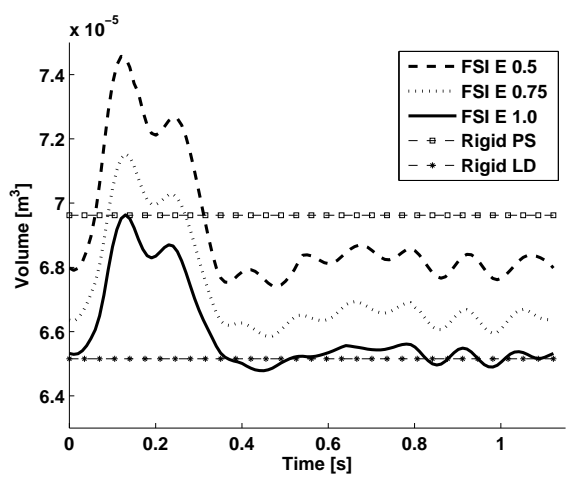

Fig. 4. The resulting volume of the fluid domain as a function of time for all five simulations. The two rigid simulations where taken from the FSI E 1.0 FSI simulation at peak systole (PS) and late diastole (LD). Notice how the volume decreases as the Young's modulus increases (FSI E $0.5<$ FSI E $0.75<$ FSI E 1.0).

\section{Results and Discussion}

In this section the velocity from the MRI measurements are compared with the FSI and rigid wall-CFD simulations to validate the simulations. Then, the pressurevolume relation of the aorta is investigated and the effect of different wall stiffnesses. In the end of the section different WSS parameters are presented.

\subsection{Velocity contours}

The in vivo velocity measured in the descending aorta (section A-A in Fig. 1) were compared with the FSI and rigid wall simulations. All velocity contours are oriented as displayed in Fig. 5.

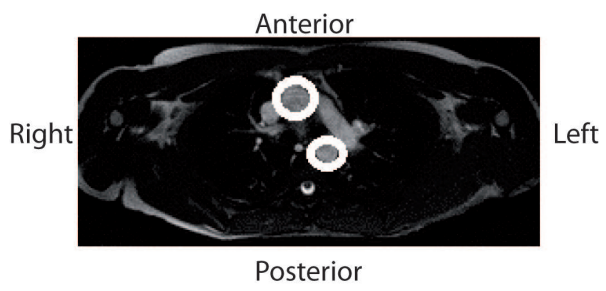

Fig. 5. A MRI cross-section view of the aorta to illustrate the orientation of the velocity contours. The ascending aorta is indicated with the upper white circle and the descending aorta is indicated with the lower circle. The MRI slice is taken at section A-A in Fig. 1.

The results were evaluated at five different times during the cardiac cycle: max acceleration, peak systole, max deceleration, early diastole and late diastole (as a reference, see Fig. 3), and are presented in Fig. 6. The left column shows the MRImeasured velocity and the view is looking from the thoracic aorta towards the aortic 
arch. The colour scale is the same for all contours and negative values corresponds to flow towards the legs, while positive values corresponds to flow towards the arch. The second, third, and fourth column shows the velocity contours obtained with the FSI simulations, and the fifth and sixth column shows the velocity contours from the two rigid wall simulations.

At max acceleration, the average velocity is approximately $0.4 \mathrm{~m} / \mathrm{s}$ in the MRI measurement, while it is about $0.1-0.15 \mathrm{~m} / \mathrm{s}$ in the FSI simulations and $0.3 \mathrm{~m} / \mathrm{s}$ in the rigid simulations. The velocity distribution is almost completely symmetric, with only a small tendency of higher velocities to the left in the FSI simulations.

At peak systole all of the velocity contours shows a very similar pattern, with a high velocity on the left side of the aorta going towards the legs (blue colour), and reverse flow on the right side going towards the arch (red colour). The backwarddirected jet is a result of recirculation that appears just after the arch, due to the curvature of the artery. The rigid models tends to have a larger area with recirculation, and it is also closer to the centre of the aorta compared to the FSI and MRI contours. The maximum velocity differs between the MRI and the simulations; the measurements show a region of velocities close to $1 \mathrm{~m} / \mathrm{s}$, while the rigid simulations have a maximum velocity close to $0.8 \mathrm{~m} / \mathrm{s}$. The FSI models have an even lower maximum velocity, around $0.75 \mathrm{~m} / \mathrm{s}$. This is due to a larger cross-sectional area during peak systole, compared to the measurements.

During max deceleration, a very good agreement between measurements and simulations is found, with a well-resolved recirculation on the right side. The downward going jet in peak systole has decreased its magnitude significantly; the average velocity of the flow going towards the legs is around $0.4 \mathrm{~m} / \mathrm{s}$ with a small region of flow having velocities of $\approx 0.5 \mathrm{~m} / \mathrm{s}$. This can also be found in the FSI models, which has an excellent agreement with the MRI in terms of resolving the backward-going recirculation and the downward-going flow. The rigid models seem to overestimate the recirculation in the right part of the profile. They also display a formation of another recirculation on the left posterior side, something that is not visible in the FSI and MRI results. This secondary recirculation is especially pronounced in the rigid geometry taken at peak systole.

At the end of systole there is almost an equal amount of flow going towards the legs as it is going towards the heart. The MRI measurement showed an average velocity of $0.01 \mathrm{~m} / \mathrm{s}$ towards the legs, while the maximum velocities were $0.26 \mathrm{~m} / \mathrm{s}$ and $0.21 \mathrm{~m} / \mathrm{s}$ to the legs and heart, respectively. The rigid models seems to move the recirculation region upwards toward the anterior side while there is a very good agreement for the FSI E 0.75 and FSI E 1.0 models with the MRI measurements.

Finally, during late diastole the flow velocity is very low, with no significant velocity contours. This is also evident by the checker board pattern in the MRI measurement, as there is almost no net flow and noise is enhanced. Normally, the signal-to-noise ratio in the MRI decreases as the flow magnitude decreases.

Overall, the qualitative agreement with measurements is very good for all mod- 
els. The backward-going jet on the right side of the aorta during peak systole to early diastole indicates a sustained axial vortex formation that disappears sometime during diastole.

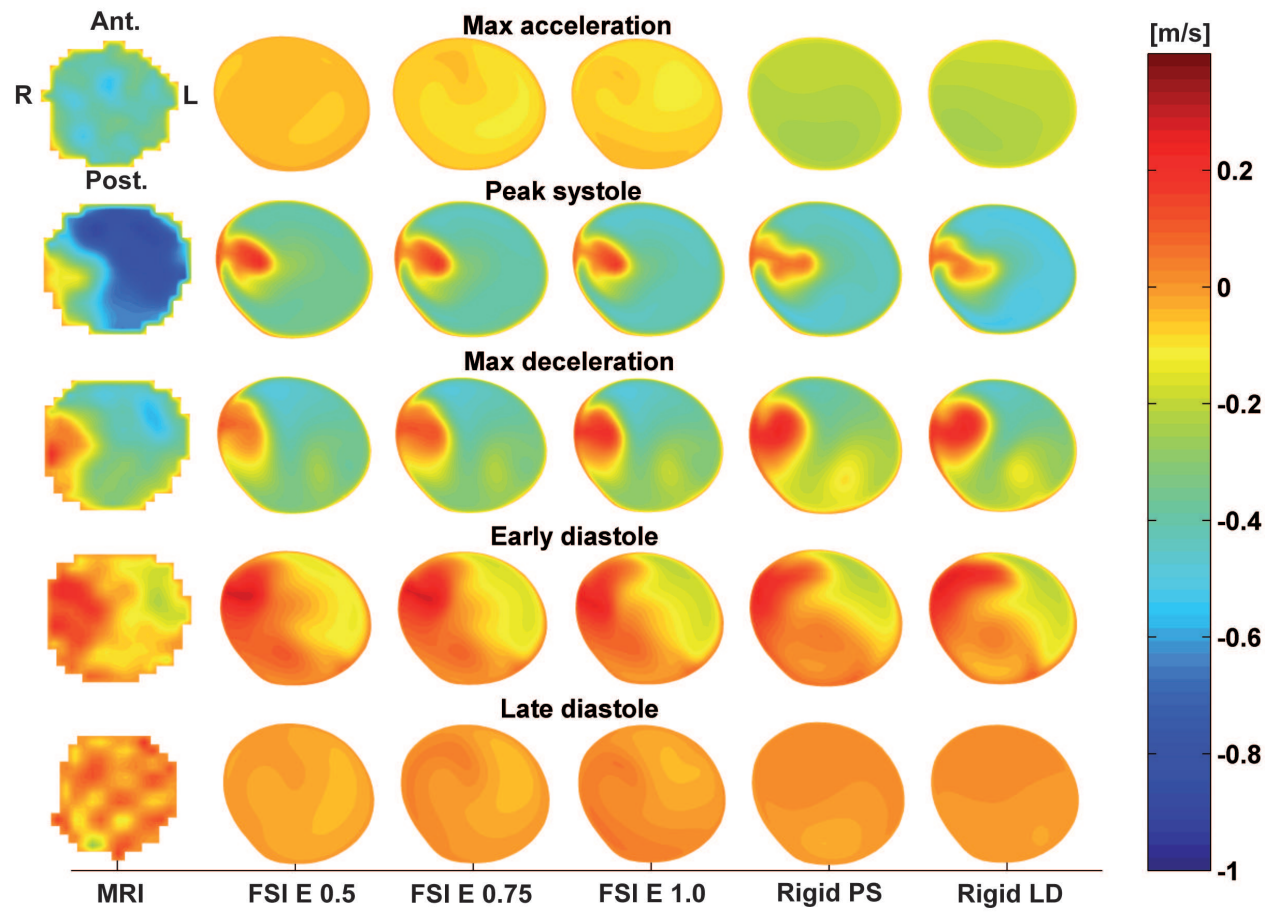

Fig. 6. Velocity contours at max acceleration, peak systole, max deceleration, early diastole and late diastole. Comparison between MRI measurements, three FSI simulations and two rigid simulations. The orientation given in the upper left corner and is the same as in Fig. 5. The velocity magnitude goes from -1 to $0.4 \mathrm{~m} / \mathrm{s}$, where negative values corresponds to flow towards the legs, and positive values flow towards the heart. In general, a good agreement is found between the measurements and the simulations, with prediction of flow reversal to the right in the descending aorta due to curvature.

\subsection{Deformation}

In order to investigate the deformation further, the area of a cross-sectional plane in the descending aorta (at section A-A in Fig. 1) was plotted against the local pressure for the three FSI models, see Fig. 7. It was found that the area could be approximated linearly to the local pressure. The slope of the curve becomes steeper with increasing Young's modulus. This was expected since a rough approximation of compliance can be defined as $C=\frac{d A}{d P}$, and as compliance is the inverse of wall stiffness, the slope of the curve should increase with increased Young's modulus.

To the right in Fig. 7 is the average pressure in the fluid domain plotted versus 
the volume of the fluid domain. A hysteresis loop is found for all three models and it is present during systole when most of the deformation occurs. Time runs counterclockwise in each curve, with peak systole occurring at max volume, followed by a short plateau where the pressure is almost constant while the volume decreases. Then the pressure starts to drop while the volume increases slightly, followed by a drop in both pressure and volume back to the start of the loop. During diastole there are small changes in both volume and pressure, but not nearly as significant as during systole. The size of the hysteresis loop was affected by the Young's modulus, as lower wall stiffness gives larger deformation.
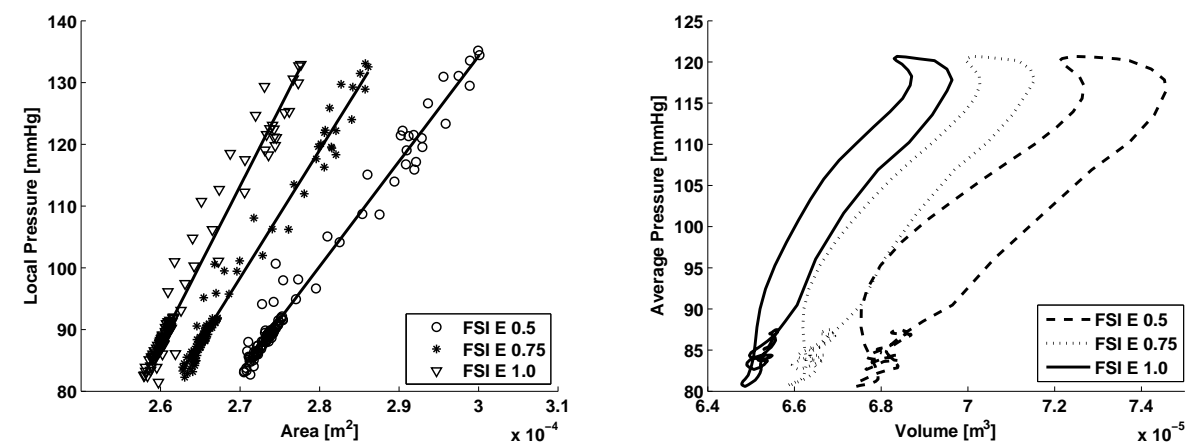

Fig. 7. Left: pressure-area relation on a cross-sectional plane in the descending aorta. The results were approximated as a linear relation, using the local pressure and area. Right: pressure-volume relation in the entire fluid domain. The hysteresis loop occurs during systole.

\subsection{Wall shear stress}

WSS is very difficult to measure in vivo, as it requires accurate information about the velocity profile close to the wall. As the spatial resolution is rather limited in the MRI measurements, no attempt was made to compute the WSS directly. Instead, it was calculated from the flow field obtained by the simulations. There might be small errors and imperfections in the geometry description compared to the actual geometry because of the limited MRI resolution. This could affect the WSS, but, as the simulations are based on the same geometry and the main goal is to investigate the differences between the models, a good estimate of the WSS can still be obtained.

\subsubsection{Time-averaged WSS}

As time-averaged WSS (TAWSS) is the shear stress the artery is subjected to over time, it is an interesting parameter to study. The WSS magnitude was averaged over the last cardiac cycle on the entire surface. As seen in Fig. 8, the time-averaged WSS pattern on all five models are similar. High values are found on the inner side of the 


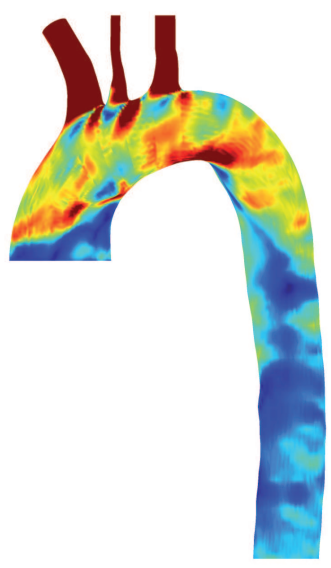

(a)

(d)

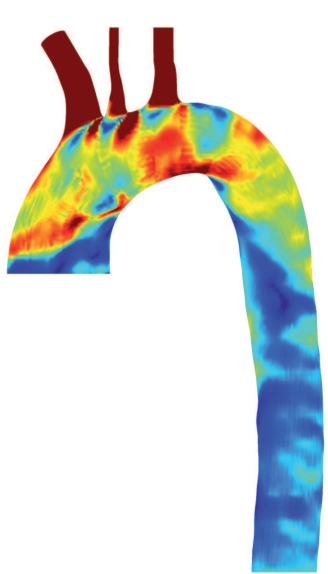

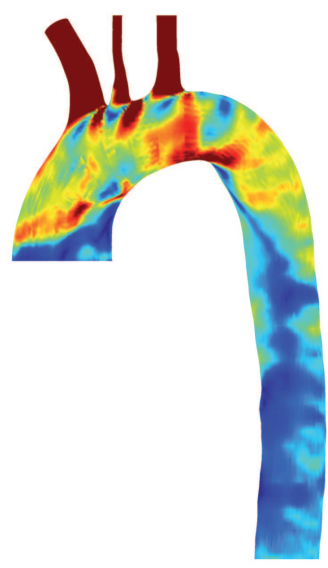

(b)

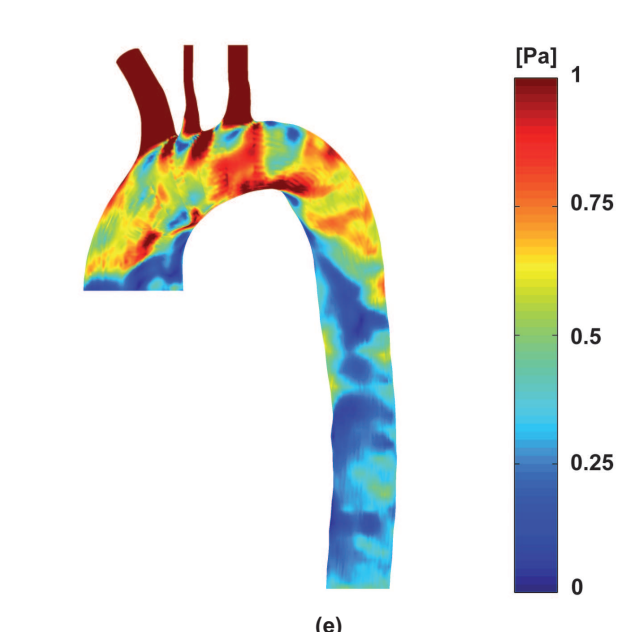

(e)

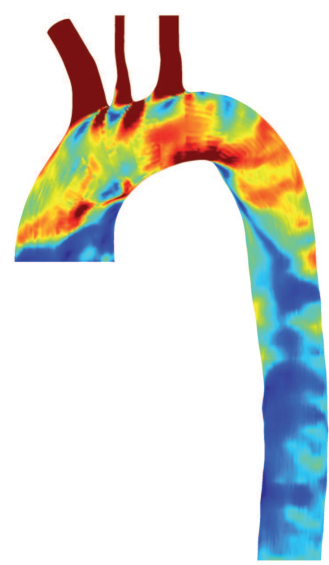

(c)

Fig. 8. Time-averaged WSS for the anterior part of the aorta. The three FSI simulations (FSI E 0.5, FSI E 0.75, and FSI E 1.0) corresponds to (a)-(c), while the Rigid PS and Rigid LD simulations corresponds to (d) and (e), respectively. Notice how similar the patterns are between the models, with only small differences in the aortic arch. The results are similar on the posterior side of the aorta and are therefore not shown.

aortic arch, which comes from the jet created during systole. As the wall stiffness increases, so does also the TAWSS in this area as seen in Figs. 8(a)-(c). The smaller arteries leaving the arch are also subjected to a high TAWSS value, as large velocity gradients are created during the acceleration phase of the pulse. In the descending and thoracic aorta the TAWSS values are low indicating that it is only the aortic 
arch and the arteries leaving the arch that are subjected to high sustained WSS values.

The rigid models are almost identical to each other with only minor differences visible in the aortic arch, indicating that there will be no significant differences in WSS with different domain volumes if the walls are rigid.

\subsubsection{Oscillatory shear index}

Another WSS parameter often used in literature is the Oscillatory Shear Index (OSI), which describes the cyclic departure of the WSS vector from its predominant axial alignment [Ku et al., 1985; He and $\mathrm{Ku}, 1996]$. It is defined as

$$
\mathrm{OSI}=0.5\left(1-\frac{\left|\frac{1}{T} \int_{0}^{T} \tau_{w} d t\right|}{\frac{1}{T} \int_{0}^{T}\left|\tau_{w}\right| d t}\right) .
$$

OSI values vary from zero to 0.5 , where zero means that the instantaneous WSS vector is aligned with the time-averaged WSS vector throughout the cardiac cycle. A value of 0.5 implies that the instantaneous WSS vector never is aligned with the time-averaged vector. OSI is insensitive to shear magnitude and must therefore be used with caution; a large OSI value can indicate a disturbed flow region with high or low WSS magnitudes. The computed OSI is seen in Fig. 9, and low values are present in the arch indicating that instantaneous WSS is directed in the timeaveraged direction. This is especially true inside the small arteries leaving the arch, as there is no backflow in those arteries. However, flow separation and recirculation occurs in the vicinity of the small arteries, and this causes the OSI to increase locally in the upper part of the arch.

The largest difference between the models is seen in the descending and thoracic aorta. The OSI patterns seem to decrease with increased wall stiffness, Figs. 9(a)(c), which can be explained by the wall deformation; the more the wall moves, the more the instantaneous WSS will deviate from the time-averaged value, increasing the OSI.

The two rigid models have OSI patterns that are very similar to the stiffest FSI model, which is reasonable as it is the model with the smallest wall deformation. In addition, there is no significant difference between the two rigid models, indicating that it is the wall motion and not the wall volume that is responsible for the change in OSI seen in the FSI models. Also, notice how the TAWSS and OSI seem to be inversely proportional to each other; a large TAWSS value yields a low OSI and vice versa.

\subsubsection{Time-resolved WSS}

To investigate how the dynamic behaviour in the FSI simulations affected the WSS, it was evaluated on a band in circumferential direction with a width of $10 \mathrm{~mm}$ in the descending aorta at section A-A. The spatial average of WSS on that location 


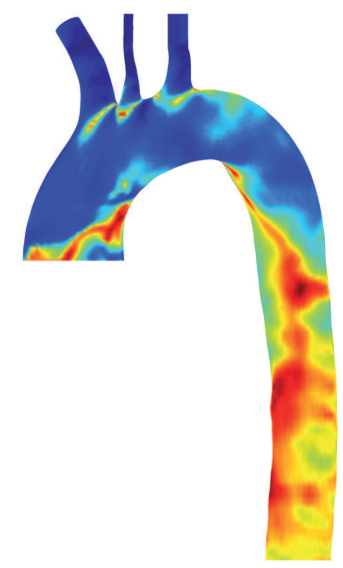

(a)

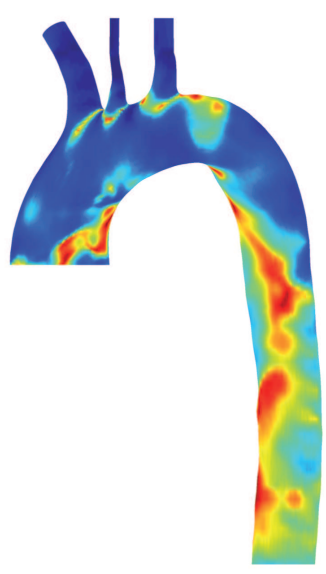

(d)

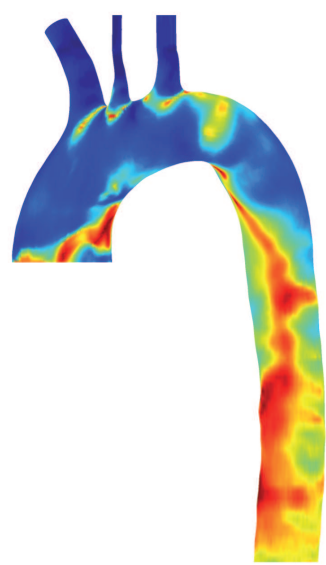

(b)

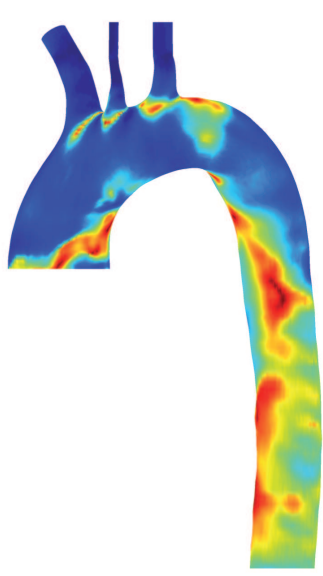

(e)

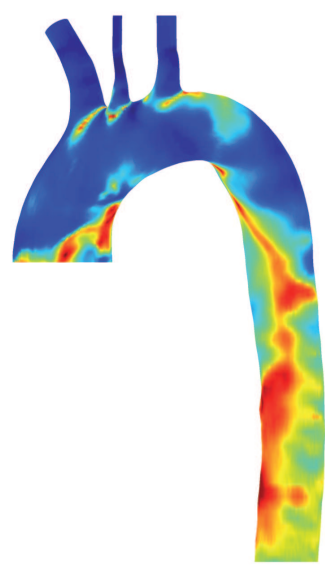

(c)

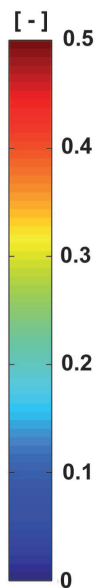

Fig. 9. OSI for the anterior part of the aorta. The three FSI simulations (FSI E 0.5, FSI E 0.75, and FSI E 1.0) correspond to Figs. (a)-(c), while the Rigid PS and Rigid LD simulations correspond to Figs. (d) and (e), respectively. Notice how similar the results are between the models, with only small differences in the descending and thoracic aorta. The OSI patterns are similar on the posterior side of the aorta and are therefore not shown.

was calculated and only the WSS during systole is shown here as there is almost no flow during diastole. In Fig. 10 the average WSS (left y-axis) is plotted against time, together with the inlet mass flow rate (right y-axis) for reference. There seems to be a delay in when the WSS increases during the acceleration phase; the rigid models have an increase in WSS about $0.05 \mathrm{~s}$ before the FSI models, which can be 


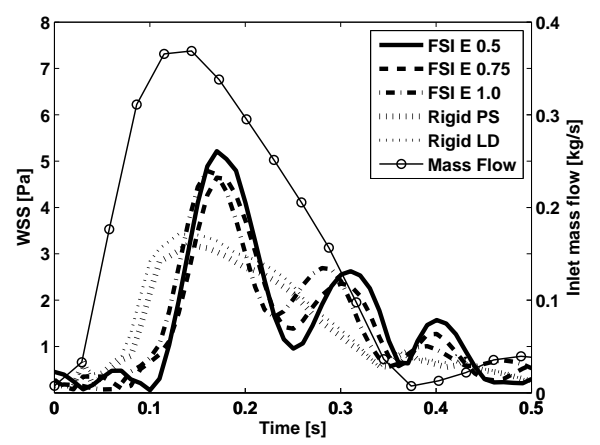

Fig. 10. The WSS during systole in a part of the descending aorta; comparison between FSI (FSI E 0.5, FSI E 0.75, and FSI E 1.0) and rigid wall models (PS and LD). The inlet mass flow is plotted as a reference (right y-axis). Notice how the WSS oscillates during the latter part of systole for the FSI models, while the rigid wall models does not show this behaviour.

explained by the dynamics introduced by the wall deformation. With a rigid wall assumption and an incompressible fluid the pressure and mass wave speeds become infinite, while with a deforming wall the wave speeds are finite. The shape of the WSS-curve in the rigid models therefore follows the shape of the mass flow pulse due to the direct influence of the inflow. On the other hand, the FSI models shows an oscillatory average WSS during the latter part of systole, which is believed to be due to the flow dynamics caused by the wall deformation. The peak value seems to depend on the wall stiffness; the stiffer the wall, the lower the WSS. However, the rigid models, which in theory has an infinite wall stiffness, has a much lower peak value. Therefore, the wall motion is believed to be important when considering instantaneous WSS.

To investigate the time-dependent WSS further, the instantaneous WSS pattern on the whole aorta was plotted at four times during the cardiac pulse for the least stiff FSI model and the smallest rigid model, see Fig. 11. The late diastole (LD) time point was excluded since there is no significant flow at that part of the cardiac pulse. Clearly, there is a big difference in instantaneous WSS patterns between a rigid wall model and a FSI model. During the max acceleration phase the WSS is much higher in the rigid model due to the infinite wave speed. In the FSI model it takes some time to transmit the inlet pulse through the domain and the WSS is therefore "lagging behind". During peak systole the FSI model show a large area with elevated WSS values in the descending and thoracic aorta which is not visible in the rigid model. During max deceleration the patterns are somewhat similar, with low WSS in the descending aorta in the rigid model while the FSI model predicts a higher value. This is also true for the early diastolic phase, but here the differences are smaller. 


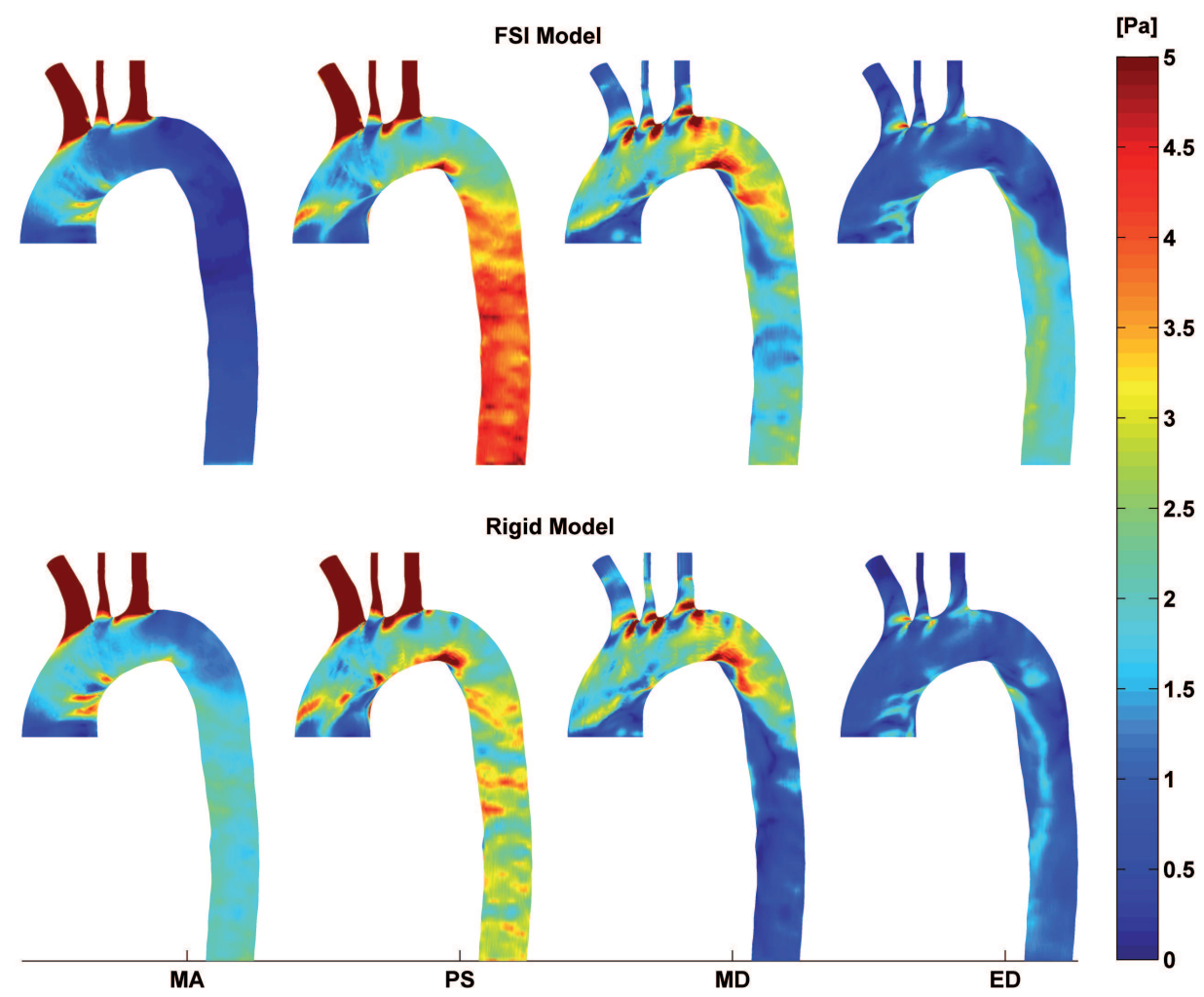

Fig. 11. Instantaneous WSS for two models at four different times during a cardiac cycle. Upper row is the FSI E 0.5 model, lower row is the Rigid LD model. MA, PS, MD, and ED stands for max acceleration, peak systole, max deceleration, and early diastole, respectively.

\section{Concluding Remarks}

This study has investigated the WSS in a MRI based subject specific human aorta, using both FSI and rigid wall models. The inlet flow profile were measured with MRI and prescribed in the ascending aorta, while an impedance pressure boundary condition was set in the thoracic aorta. Velocity contours in the descending aorta were found to be in very good agreement with MRI measurements, with prediction of flow reversal on the inner side in the descending aorta. Small differences in TAWSS and OSI could be found between the FSI and rigid wall models, but when regarding instantaneous WSS large differences were found. Therefore, if the instantaneous WSS is important it is necessary to consider a FSI simulation that can account for the deformation of the arterial wall. 


\section{Acknowledgements}

This work was supported by grants from the Swedish research council, VR 2007-4085 and VR 2010-4282. The Swedish National Infrastructure for Computing (SNIC) is acknowledged for computational resources provided by the National Supercomputer Centre (NSC) under grant No. SNIC022/09-11. Dr. Tino Ebbers at the Department of Medicine and Care at Linköpings University is acknowledged for the MRI measurements. This work has been conducted in collaboration with the Center for Medical Image Science and Visualization (CMIV, http://www.cmiv.liu.se/) at Linköping University, Sweden. CMIV is acknowledged for provision of financial support and access to leading edge research infrastructure.

\section{References}

ANSYS Inc. [2010a] ANSYS CFX-Solver Modeling Guide, 275 Technology Drive, Canonsburg, PA 15317, USA.

ANSYS Inc. [2010b] ANSYS ICEM CFD User Guide, 275 Technology Drive, Canonsburg, PA 15317, USA.

Bathe, M., Kamm, R. D. and Kamm, R. D. [1999] A fluid-structure interaction finite element analysis of pulsatile blood flow through a compliant stenotic artery, Journal of Biomechanical Engineering 121, 361-369.

Beulen, B., Rutten, M. and van de Vosse, F. [2009] A time-periodic approach for fluidstructure interaction in distensible vessels, Journal of Fluids and Structures 25(5), $954-966$.

Cheng, R., Lai, Y. G. and Chandran, K. B. [2003] Two-dimensional fluid-structure interaction simulation of bileaflet mechanical heart valve flow dynamics, Journal of Heart Valve Disease 12, 772-780.

Cheng, R., Lai, Y. G. and Chandran, K. B. [2004] Three-dimensional fluid-structure interaction simulation of bileaflet mechanical heart valve flow dynamics, Annals of Biomedical Engineering 32, 1471-1483.

Dahl, S. K., Vierendeels, J., Degroote, J., Annerel, S., Hellevik, L. R. and Skallerud, B. [2010] FSI simulation of asymmetric mitral valve dynamics during diastolic filling, Computer Methods in Biomechanics and Biomedical Engineering, 1.

Dempere-Marco, L., Oubel, E., Castro, M., Putman, C., Frangi, A. and Cebral, J. [2006] CFD analysis incorporating the influence of wall motion: application to intracranial aneurysms, Medical Image Computing and Computer-Assisted Intervention 9, 438445.

Gao, F., Guo, Z., Sakamoto, M. and Matsuzawa, T. [2006a] Fluid-structure interaction within a layered aortic arch model, Journal of Biological Physics 32, 435-454.

Gao, F., Watanabe, M. and Matsuzawa, T. [2006b] Stress analysis in a layered aortic arch model under pulsatile blood flow, BioMedical Engineering OnLine 5, 25.

Grant, B. J. and Paradowski, L. J. [1987] Characterization of pulmonary arterial input impedance with lumped parameter models, American Journal of Physiology 252, H585-593.

He, X. and Ku, D. N. [1996] Pulsatile flow in the human left coronary artery bifurcation: average conditions, Journal of Biomechanical Engineering 118, 74-82.

Heiberg, E., Sjogren, J., Ugander, M., Carlsson, M., Engblom, H. and Arheden, H. [2010] Design and validation of Segment-freely available software for cardiovascular image analysis, BMC Medical Imaging 10, 1. 
Irace, C., Cortese, C., Fiaschi, E., Carallo, C., Farinaro, E. and Gnasso, A. [2004] Wall shear stress is associated with intima-media thickness and carotid atherosclerosis in subjects at low coronary heart disease risk, Stroke 35, 464-468.

Isaksen, J. G., Bazilevs, Y., Kvamsdal, T., Zhang, Y., Kaspersen, J. H., Waterloo, K., Romner, B. and Ingebrigtsen, T. [2008] Determination of wall tension in cerebral artery aneurysms by numerical simulation, Stroke 39, 3172-3178.

Jeltsch, M., Klass, O., Klein, S., Feuerlein, S., Aschoff, A. J., Brambs, H. J. and Hoffmann, M. H. [2009] Aortic wall thickness assessed by multidetector computed tomography as a predictor of coronary atherosclerosis, International Journal of Cardiovascular Imaging 25, 209-217.

Jin, S., Oshinski, J. and Giddens, D. P. [2003] Effects of wall motion and compliance on flow patterns in the ascending aorta, Journal of Biomechanical Engineering 125, $347-354$.

Kelly, S. C. and O'Rourke, M. J. [2010] A two-system, single-analysis, fluid-structure interaction technique for modelling abdominal aortic aneurysms, Proceedings of the Institution of Mechanical Engineers. Part H, Journal of engineering in medicine 224, 955-969.

Kenner, T. [1989] The measurement of blood density and its meaning, Basic Research in Cardiology 84, 111-124.

Ku, D. N., Giddens, D. P., Zarins, C. K. and Glagov, S. [1985] Pulsatile flow and atherosclerosis in the human carotid bifurcation. Positive correlation between plaque location and low oscillating shear stress, Arteriosclerosis 5, 293-302.

Li, Z. and Kleinstreuer, C. [2005a] Blood flow and structure interactions in a stented abdominal aortic aneurysm model, Medical Engineering $\& 3$ Physics 27, 369-382.

Li, Z. and Kleinstreuer, C. [2005b] Fluid-structure interaction effects on sac-blood pressure and wall stress in a stented aneurysm, Journal of Biomechanical Engineering 127, 662-671.

Malek, A. M., Alper, S. L. and Izumo, S. [1999] Hemodynamic shear stress and its role in atherosclerosis, Journal of the American Medical Association 282, 2035-2042.

McGregor, R. H., Szczerba, D. and Szekely, G. [2007] A multiphysics simulation of a healthy and a diseased abdominal aorta, Medical Image Computing and Computer-Assisted Intervention 10, 227-234.

Moore, J. E., Xu, C., Glagov, S., Zarins, C. K. and Ku, D. N. [1994] Fluid wall shear stress measurements in a model of the human abdominal aorta: oscillatory behavior and relationship to atherosclerosis, Atherosclerosis 110, 225-240.

Scotti, C. M., Jimenez, J., Muluk, S. C. and Finol, E. A. [2008] Wall stress and flow dynamics in abdominal aortic aneurysms: finite element analysis vs. fluid-structure interaction, Computer Methods in Biomechanics and Biomedical Engineering 11, 301-322.

Shahcheraghi, N., Dwyer, H. A., Cheer, A. Y., Barakat, A. I. and Rutaganira, T. [2002] Unsteady and three-dimensional simulation of blood flow in the human aortic arch, Journal of Biomechanical Engineering 124, 378-387.

Simon, H. A., Ge, L., Borazjani, I., Sotiropoulos, F. and Yoganathan, A. P. [2010] Simulation of the three-dimensional hinge flow fields of a bileaflet mechanical heart valve under aortic conditions, Annals of Biomedical Engineering 38, 841-853.

Stergiopulos, N., Westerhof, B. E. and Westerhof, N. [1999] Total arterial inertance as the fourth element of the windkessel model, American Journal of Physiology 276, H81-88.

Svensson, J., Gardhagen, R., Heiberg, E., Ebbers, T., Loyd, D., Lanne, T. and Karlsson, M. [2006] Feasibility of patient specific aortic blood flow cfd simulation, in R. Larsen, 
M. Nielsen and J. Sporring (eds.), Medical Image Computing and Computer-Assisted Intervention, Lecture Notes in Computer Science, Vol. 4190, pp. 257-263.

Takizawa, K., Moorman, C., Wright, S., Christopher, J. and Tezduyar, T. [2010] Wall shear stress calculations in spacetime finite element computation of arterial fluidstructure interactions, Computational Mechanics 46, 31-41.

Tan, F., Torii, R., Borghi, A., Hohiaddin, R., Wood, N. and XU, X. [2009] Fluid-structure interaction analysis of wall stress and flow patterns in a thoracic aneurysm, International Journal of Applied Mechanics 1, 179-199.

Wells, R. E. and Merril, E. W. [1962] Influence of flow properties of blood upon viscosityhematocrit relationships, Journal of Clinical Investigation 41, 1591-1598.

Westerhof, N., Lankhaar, J. W. and Westerhof, B. E. [2009] The arterial Windkessel, Medical \& Biological Engineering \& Computing 47, 131-141.

Wolters, B. J., Rutten, M. C., Schurink, G. W., Kose, U., de Hart, J. and van de Vosse, F. N. [2005] A patient-specific computational model of fluid-structure interaction in abdominal aortic aneurysms, Medical Engineering \& Physics 27, 871-883.

Xenos, M., Rambhia, S. H., Alemu, Y., Einav, S., Labropoulos, N., Tassiopoulos, A., Ricotta, J. J. and Bluestein, D. [2010] Patient-based abdominal aortic aneurysm rupture risk prediction with fluid structure interaction modeling, Annals of Biomedical Engineering 38, 3323-3337. 